\title{
Patient satisfaction in the long-term effects of Eustachian tube balloon dilation is encouraging
}

\section{Luukkainen, Veera}

2018-02

Luukkainen , V , Vnencak, M , Aarnisalo , A A , Jero , J \& Sinkkonen , S T 2018 , ' Patient satisfaction in the long-term effects of Eustachian tube balloon dilation is encouraging ' , Acta Oto-Laryngologica , vol. 138 , no. 2 , pp. 122-127 . https://doi.org/10.1080/00016489.2017.1384568

http://hdl.handle.net/10138/313217

https://doi.org/10.1080/00016489.2017.1384568

acceptedVersion

Downloaded from Helda, University of Helsinki institutional repository.

This is an electronic reprint of the original article.

This reprint may differ from the original in pagination and typographic detail.

Please cite the original version. 
1 Patient satisfaction in the long-term effects of Eustachian tube balloon

2 dilation is encouraging

$4 \quad{ }^{I}$ Veera Luukkainen, ${ }^{I}$ Matej Vnencak, ${ }^{l}$ Antti A. Aarnisalo, ${ }^{1,2}$ Jussi Jero,

$5 \quad{ }^{\text {SSaku T. Sinkkonen }}$

$7 \quad$ IDepartment of Otorhinolaryngology, Head and Neck Center, Helsinki University

8 Hospital and University of Helsinki, Helsinki, Finland.

9

${ }^{2}$ Department of Otorhinolaryngology - Head and Neck Surgery, Turku University

10 Hospital and University of Turku, Turku, Finland.

11

12 Veera Luukkainen: Helsinki University Hospital, Head and Neck Center, Dept.

13 Otorhinolaryngology, POB 263, FIN-00029 HUH, FINLAND; Tel: +358-50-4279331;

14 Fax: +358-9-47175047; e-mail: veera.luukkainen@hus.fi. Corresponding author.

15 Matej Vnencak: Helsinki University Hospital, Head and Neck Center, Dept.

16 Otorhinolaryngology, POB 263, FIN-00029 HUH, FINLAND; e-mail:

17 matej.vnencak@yahoo.com.

Antti A. Aarnisalo: Helsinki University Hospital, Head and Neck Center, Dept.

19

Otorhinolaryngology, POB 263, FIN-00029 HUH, FINLAND; e-mail:

antti.aarnisalo@hus.fi.

21 Jussi Jero: Helsinki University Hospital, Head and Neck Center, Dept.

22 Otorhinolaryngology, POB 263, FIN-00029 HUH, FINLAND; e-mail: jussi.jero

23 @hus.fi. 
1 Saku T. Sinkkonen: Helsinki University Hospital, Head and Neck Center, Dept.

2 Otorhinolaryngology, POB 263, FIN-00029 HUH, FINLAND; e-mail:

3 saku.sinkkonen@hus.fi.

4

5

6 Word count: 3035 .

7

8 
1 Patient satisfaction in the long-term effects of Eustachian tube balloon

\section{2 dilation is encouraging}

3

Object: Investigate the long-term effects of balloon Eustachian tuboplasty (BET) from patient's perspective and to discover which symptoms of Eustachian tube dysfunction (ETD) benefit the most from BET.

Method: We designed a retrospective postal questionnaire based on the sevenitem ETD questionnaire (ETDQ-7). Our questionnaire covered the severity of present ETD symptoms in comparison with the preoperative situation, the severity of current overall ear symptoms, and possible surgical interventions after BET. Forty-six patients treated in our institution between 2011 and 2013 fulfilled the inclusion criteria and $74 \%$ (34 patients; total 52 ears treated with BET) returned the questionnaire with a mean follow-up time of 3.1 years (range 1.8-4.6 years).

Results: Pain in the ears, feeling of pressure in the ears, and feeling that ears are clogged had reduced in $75 \%$ of the ears that suffered from these symptoms preoperatively. Seventy-seven percent of all the responders felt that their overall ear symptoms were reduced. Altogether, 79\% of all the patients stated that they would undergo BET again if their ear symptoms returned to the preoperative level.

Conclusion: Patient satisfaction in the long-term effects of BET is encouraging. These results may help clinicians in preoperative patient selection and counselling.

Keywords: Eustachian tube dysfunction, balloon dilation, questionnaire, patient satisfaction 


\section{Methods} [2-4].

\section{Introduction}

The Eustachian tube (ET) has a central role in aeration of the middle ear. Therefore, prolonged ET dysfunction (ETD) can lead to chronic ear diseases [1, 2]. One method aiming to manage ETD and thereby prevent chronic ear diseases is ET balloon dilation (balloon Eustachian tuboplasty, BET). How to most accurately evaluate the effect of BET is still unclear. Clinical findings, tubomanometry, ET score (ETS), its extension ETS-7, and the seven-item ETD questionnaire (ETDQ-7) are some examples of methods available to help in the diagnostics of ETD and in assessing the effects of its treatment

Studies on the short-term effect of BET suggest that the procedure is effective and safe [5-7]. Aural fullness, earache, pressure in the ear, hearing impairment, and vertigo have been reported to improve in the short term. [5, 7]. Two long-term follow-up studies showed significant improvement in ETS and overall ear symptoms at two years, and in clinical findings and the ability to perform the Valsalva maneuver after follow-up of 2.5 years $[1,8]$. Another study stated that 8 patients were followed for at least 34 months and that seven of them reported persistent improvement [9]. The long-term studies left specific symptoms that improved undefined. Therefore, we now concentrated on longterm patient-reported outcome with a questionnaire covering symptoms of ETD.

\section{Ethical approval}

The study was approved by the Ethics Committee of Department of Surgery, Helsinki University Hospital, Helsinki, Finland (reg. no. 60/13/03/02/2015). The patients gave 
1 informed consent before enrolment in the study. The study was performed in accordance

2 with the Declaration of Helsinki.

3

4

\section{Patients}

6 We retrospectively identified 51 consecutive patients treated with BET in our department from 2011 to 2013. Simultaneous ear operations, except tympanocentesis (2 patients) or tympanostomy tube insertion (3 patients), lead to exclusion, as did previous Eustachian

9 tube operations. Forty-six patients met the inclusion criteria and received a postal 10 questionnaire, and 34 (74\%) returned it. ET dysfunction type as defined in the consensus

11 statement [10] was of dilatory type in 31 of the responders and baro-challenge-induced

12 in three responders.

Preoperative studies included full otorhinolaryngological examination including

14 otomicroscopy and nasoendoscopy (or anterior and posterior rhinoscopy). Tympanic

15 membrane was retracted or tympanometry showed negative pressure in $76 \%$ of the

16 responders. The nasopharynx and ET openings were normal in 50\%. In 35\%, the mucosa

17 was swollen. In $6 \%$, the Eustachian tube openings were especially narrow or scarred from

18 previous adenotomy. In $9 \%$, the condition was undefined in the medical charts. Two

19 patients had small nasal polyps.

After BET, 25 patients required no further interventions whereas nine patients

21 underwent additional operations because their ear symptoms persisted. Six patients

22 required tympanostomy tubes once or repeatedly during the follow-up. One patient

23 received radical mastoidectomy because previously operated cholesteatoma recurred.

24 Other two patients previously operated for cholesteatoma experienced no recurrence 
1 during the follow-up, and their ear symptoms required no further interventions after BET. One patient, with history of recurring otitis media and myringostapedopexy, received tympanoplasty. In one patient, scarred tubal opening on the left prevented BET to that side but the operation was successful to the right. Later, he received bilateral BET but required repeated bilateral tympanocenteses even after that. No symptom or diagnosis clearly distinguished the patients who needed additional operations from those who received only BET. Diagnoses were chronic secretory otitis media (64\% of those that received only BET and $78 \%$ of those that underwent additional interventions), Eustachian

9 tube dysfunction (28\% and $8 \%$ ), and chronic otitis media (8\% and $0 \%)$.

Details on demography, preoperative clinical findings, treatment, and possible

11 further interventions were recorded from the medical charts.

\section{Questionnaire}

14 The postal questionnaire was modified from ETDQ-7, which covers the severity of 15 specific ear symptoms suggestive of ETD from the previous month [4, 9]. The ETDQ-7

16 is the only disease-specific questionnaire validated for diagnostics of ETD and for 17 evaluating the outcome of its treatment. First, the questions from the English version of 18 ETDQ-7 were translated to Finnish and Swedish (the official languages of Finland). As 19 detailed information of the symptoms was not gathered preoperatively, we modified the questions so that we asked the patients if specific ear symptoms covered in the ETDQ-7

21 were currently clearly milder, somewhat milder, the same, somewhat worse, or clearly worse compared to the preoperative situation. In addition to the symptom specific questions based on ETDQ-7 we composed additional questions. We asked if the patients could perform Valsalva maneuver better than before the operation. The maneuver was 
1 explained to the patients in the questionnaire. We also asked if swallowing releases pressure in the ears now better than preoperatively, how the patients would rate the severity of their overall ear symptoms now, and how disturbing their current ear symptoms are. The questionnaire also covered possible visits to a doctor and procedures

5 done after BET in order to treat ear symptoms. The questionnaire is provided in

6 Additional Files 1-3 (Finnish version in Additional File 1, Swedish version in Additional

7 File 2, and their translation to English in Additional File 3).

\section{Surgical procedure}

10 The patients were under general anesthesia, and cocaine-adrenaline solution in small

11 absorbent cotton pads provided nasal nerve block anesthesia. The balloon dilation was 12 carried out with sinus balloon dilation catheter, $7 \mathrm{~mm}$ x $16 \mathrm{~mm}$ Acclarent Relieva solo

13 (Acclarent, Inc., Menlo Park, CA, USA). Rigid nasoendoscopes with diameters of 3-4 $14 \mathrm{~mm}$ and angles from $0^{0}$ to $45^{\circ}$ allowed visual control. The endoscope was inserted 15 through the ipsilateral nostril if the nasal anatomy allowed it. If not, it was inserted 16 through the contralateral nostril. When properly inserted to the Eustachian tube, the 17 balloon was inflated once to 10-12 atm for two minutes or twice to $10-12$ atm for one 18 minute. Experienced ENT doctors performed the operations with the two-hands technique. No immediate or late complications occurred.

\section{$21 \quad$ Statistics}

22 The differences in prevalences of the symptoms and in the effect of BET on different 23 symptoms in Table 2 were calculated using logistic regression and Fisher's exact test 
1 (SAS software, SAS Institute Inc., Cary, NC, USA). Fischer's exact test was used with

2 Table 3.

3

\section{Results}

5 No differences existed in gender, age, or diagnosis between all the 46 patients meeting

6 the inclusion criteria and the 34 responders (Table 1). The follow-up time for the responders was $3.1 \pm 0.1$ years (mean \pm SEM, range 1.8-4.6).

The most common preoperative symptoms among all the responders $(n=34,52$

9 ears, Table 2a), were feeling that ears are clogged (96\% of treated ears) and muffled

10 hearing $(92 \%)$. Other common symptoms were ear symptoms during a cold (77\%),

11 crackling or popping sounds in the ears (77\%), and pressure in the ears (73\%). There was

12 no statistically significant difference $(p=0.1584)$ between the prevalences of feeling that

13 ears are clogged and muffled hearing. However, they both had significantly higher

14 prevalence than any other symptoms $(p \leq 0.0390)$. Patients with pain in the ears seemed

15 to benefit the most from the procedure as pain reduced in $81 \%$ of preoperatively affected

16 ears. Pain in the ears as well as pressure in the ears (improvement in 79\%), feeling that

17 ears are clogged (76\%), and ear symptoms during a cold (66\%) improved significantly

18 more $(p \leq 0.0346)$ than ringing in the ears (improvement in $40 \%)$ and being able to release

19 pressure in the ears by swallowing (39\%). Ability to perform Valsalva maneuver improved in 50\%, which was significantly less $(p \leq 0.0297)$ than improvement of pain in

21 the ears, pressure in the ears, and clogged feeling in the ears.

Among those who underwent only BET ( $n=25,38$ ears), the most common 23 symptoms were feeling that ears are clogged (95\%) and muffled hearing (89\%) (Table 24 2b). The prevalence of clogged feeling was significantly higher $(p \leq 0.0364)$ than that of 
1 any other symptom except muffled hearing. The difference between the prevalences of muffled hearing and any other symptom than clogged feeling or ear symptoms during a cold was also statistically significant $(p \leq 0.0493)$. At least $70 \%$ of the ears improved regarding feeling of pressure, ear symptoms during a cold, clogged feeling, pain, and

5 muffled hearing. Those who had trouble releasing pressure in the ears by swallowing benefitted the least from BET in this group as only $48 \%$ were better at it at the end of the follow-up than preoperatively. Valsalva maneuver improved only in $57 \%$ of the ears. Ear symptoms during a cold improved significantly more than ringing in the ears and

9 swallowing releasing pressure in the ears $(83 \%, 50 \%$ and $48 \%$, respectively, $p \leq 0.0155)$.

10 Feeling of pressure in the ears improved significantly more than swallowing releasing

11 pressure $(87 \%$ and $48 \%$, respectively, $p=0.0101)$. Other differences in improvement of 12 different symptoms were statistically insignificant in this group. In the group that underwent additional procedures after BET $(n=9,14$ ears), every 14 ear had preoperatively suffered from feeling that ears are clogged, muffled hearing, and pressure in the ears (Table 2c). However, the symptom that reduced the most in this group, in $89 \%$ of the preoperatively symptomatic ears, was pain in the ears. This decrease and the reduction of clogged feeling in the ears $(71 \%)$ were significantly better results than improvement in Valsalva maneuver (28\%), releasing pressure in the ears by swallowing (20\%), ear symptoms during a cold (18\%), or ringing in the ears $(0 \%)(p \leq 0.0423)$. Pressure in the ears improved in $64 \%$ which was significantly more than improvement of Valsalva maneuver, ear symptoms during a cold, or ringing in the ears $(p \leq 0.0410)$. When asked about the change in overall ear symptoms, $77 \%$ of all treated ears 23 had now less symptoms than preoperatively as $10 \%$ were asymptomatic, $42 \%$ had clearly less and 25\% somewhat less symptoms than preoperatively (Table 3). Those who felt they 
1 benefitted from the procedure (symptoms less than preoperatively, 77\%) significantly outnumbered those whose overall symptoms showed no improvement $(23 \%)(p=$

3 0.0060). Of those patients who only underwent BET, symptoms had disappeared or

4 reduced in $90 \%$ whereas of those who required additional interventions only $43 \%$ had

5 reduced symptoms and none was asymptomatic.

When asked how disturbing the current ear symptoms were, $67 \%$ of all the responders reported that their current symptoms disturbed them not at all, very little, or

8 little (Table 4). Of those that underwent only BET, $8 \%$ were asymptomatic, $36 \%$

9 experienced very little disturbance, and $32 \%$ little disturbance. This amounts to $76 \%$ of

10 less disturbing ear symptoms in this group. However, in the group that had undergone

11 additional procedures, 55\% experienced more than little disturbance from their ear

12 symptoms suggesting overall more treatment resistant symptoms in this group. Finally, we asked the patients whether they, with their current knowledge of BET

14 and its effects on them personally, would be willing to undergo BET again if their 15 symptoms returned to the preoperative level. In that situation, $79 \%$ of all the responders would choose BET again (Table 5). Of those who received only BET, 84\% would undergo BET again. Of those who required additional interventions, $67 \%$ would undergo

18 BET again.

\section{Discussion}

21 The long-term effect of BET seems satisfactory as $77 \%$ of the treated ears were less symptomatic after the follow-up than preoperatively. Symptoms reduced in $90 \%$ of the group that only underwent the initial BET. BET best alleviated pain in the ears, feeling of pressure in the ears, and feeling that ears are clogged as each of these symptoms 
1 improved in over $75 \%$ of all the affected ears. Ringing in the ears and the ability to release pressure in the ears by swallowing responded the least to BET. The Valsalva maneuver became easier in only $50 \%$ of all treated ears and only in $57 \%$ of those who required no further interventions after BET. Of all the responders, $79 \%$ would undergo BET again, if their symptoms returned to the preoperative level. Even though only $43 \%$ of those who needed additional operations were less symptomatic at the end of the follow-up than preoperatively, $67 \%$ of them would undergo BET again if their ear symptoms returned to the preoperative level. That might suggest that the positive effect of BET might have been

9 stronger earlier and the symptoms might have been returning towards the end of the follow-up. However, this might also reflect that the patients hope that reoperation would have a better effect than the first operation.

Even though the postal survey return rate, $74 \%$, is good, those who experienced no benefit from the procedure might have failed to respond rendering our results as overly 14 optimistic. However, no differences existed in demographics between all the treated patients and the responders (Table 1) suggesting that our sample population is representative. As this was a retrospective questionnaire study, there was no baseline assessment, and preoperative data in the medical charts was incomplete regarding the symptoms listed in our questionnaire. Therefore, it was up to the patients to remember the preoperative situation. There were no routine visits at the clinic at the time the patients returned the questionnaire, and thus no objective information was obtained. Nonetheless, in this particular setting we were interested in the patient's perspective on the long-term effect of BET. It is noteworthy, that the ETDQ-7 is validated in English, and the Finnish and Swedish modifications used in this study have not been validated. Also, the English ETDQ-7 is validated for assessing ETD-related symptoms at a given time period and for 
1 evaluating treatment outcome when the questionnaire is used both pre- and postoperatively. In this study we used the modified questionnaire with no systematic pretreatment symptom assessment. For that reason, it is not certain that the questionnaire

$4 \quad$ used in this study has validity for the purpose. Schröder and co-workers [1] reported that $73 \%$ of 89 patients were free of symptoms or had less symptoms two years after BET. In our study, $77 \%$ of the 52 BETtreated ears were asymptomatic or had reduced symptoms after mean follow-up of 3.1 years. Wanscher and Svane-Knudsen [7] found that, at two months, Valsalva maneuver

9 had improved in $66 \%$ and earache in 55\%. In our study, the Valsalva maneuver improved in $50 \%$ and earache in $81 \%$. Silvola and colleagues [8] reported that after mean follow-

11 up of 2.5 years, the Valsalva maneuver had clearly improved in $80 \%$. However, in their 12 study, the examiner evaluated the maneuver whereas in ours and one by Wanscher and 13 Svane-Knudsen the patients themselves evaluated it. Gürtler and colleagues [5] found

14 that, at three months, feeling of pressure had disappeared in $75 \%$ of preoperatively 15 symptomatic patients and hearing impairment in $44 \%$ but tinnitus in none. In our study, 16 pressure clearly improved in $42 \%$, muffled hearing in $23 \%$, and ringing in the ears in 17 20\%. Catalano and co-workers treated 70 patients (100 ears) with BET [9]. Mean follow18 up time was 30.3 weeks, and eight patients were followed for at least 34 months. They 19 registered feeling of fullness in the ear, pressure, pain, tolerance to flying, and objective 20 findings in tympanic membrane at each visit. Of those eight patients that had the longest 21 follow-up, seven reported persistent improvement. However, it is unclear which 22 symptoms were relieved and if the report of persistent improvement was purely subjective 23 or if supporting objective findings were present. McCoul and Anand performed BET and 24 partial inferior turbinectomy on 22 patients (total 35 cases) with a 6-month follow-up 
1 [11]. They also performed simultaneous submucous resection of the nasal septum in 15

2 cases while 17 cases received simultaneous endoscopic sinus surgery. Retraction of

3 tympanic membrane was present in 33 cases (94\%) preoperatively and healed in all of

4 them during the follow-up. Preoperatively, tympanogram was abnormal in all cases but

5 postoperatively only in one case with tympanic membrane perforation. In our study,

6 tympanic membrane retraction or negative pressure in the tympanogram was

\section{preoperatively present in $76 \%$. In addition to clinical findings, McCoul and Anand} evaluated the response to BET by ETDQ-7, which the patients completed preoperatively and at every follow-up visit. In addition to ETDQ-7, patients were asked at every visit whether their overall ETD symptoms had reduced since BET. ETDQ-7 improved significantly at every follow-up point, and it had a significant association to patient's evaluation of overall symptom reduction. Our study had a different questionnaire than the original ETDQ-7 and it has no similar validity. However, based on the findings by McCoul and Anand, it could be suggested that as $77 \%$ of patients evaluated improvement in their overall ear symptoms since BET, they probably would have shown decrease in ETDQ-7 after the follow-up if ETDQ-7 had been used in its original form pre- and postoperatively.

8 The results of this study add to the evidence that BET is a simple and safe procedure with significant subjective long-term benefits to the patients [1, 8-9]. We now know which specific symptoms are most likely to improve in long term. These findings, together with the results of other studies, help clinicians in preoperative patient selection and counselling. However, more prospective studies on validated ETDQ-7 together with objective outcome measures are needed to support the findings of this study. 


\section{Disclosure statement}

2 The authors report no conflicts of interest.

3

4

6 


\section{REFERENCES}

2

3 1. Schröder S, Lehmann M, Ebmeyer J, Upile T, Sudhoff H. Balloon Eustachian

4 tuboplasty: a retrospective cohort study. Clin Otolaryngol. 2015; 40:629-638.

5 2. Schröder S, Lehmann M, Korbmacher D, Sauzet O, Sudhoff H, Ebmeyer J. Evaluation

6 of tubomanometry as a routine diagnostic tool for chronic obstructive Eustachian tube

7 dysfunction. Clin Otolaryngol. 2015; 40:691-697.

8 3. Schröder S, Lehmann M, Sauzet O, Ebmeyer J, Sudhoff H. A novel diagnostic tool for

9 chronic obstructive eustachian tube dysfunction - the Eustachian tube score.

10 Laryngoscope. 2015; 125:703-708.

11 4. McCoul ED, Anand VK, Christos PJ. Validating the clinical assessment of Eustachian

12 tube dysfunction: The Eustachian Tube Dysfunction Questionnaire (ETDQ-7).

13 Laryngoscope. 2012; 122:1137-1141.

14 5. Gürtler N, Husner A, Flurin H. Balloon Dilation of the Eustachian Tube: Early

15 Outcome Analysis. Otol Neurotol. 2014; 36:437-443.

16 6. Poe DS, Silvola J, Pyykkö I. Balloon dilation of the cartilaginous Eustachian tube.

17 Otolaryngol Head Neck Surg. 2011; 144:563-569.

18 7. Wanscher JH, Svane-Knudsen V. Promising results after balloon dilatation of the

19 Eustachian tube for obstructive dysfunction. Dan Med J. 2014; 61:A4818.

20 8. Silvola J, Kivekäs I, Poe DS. Balloon Dilation of the Cartilaginous Portion of the 21 Eustachian Tube. Otolaryngol Head Neck Surg. 2014; 151:125-130.

22 9. Catalano PJ, Jonnalagadda S, Yu VM. Balloon catheter dilatation of Eustachian tube:

23 a preliminary study. Otol Neurotol. 2012; 33:1549-52. 
1 10. Schilder AG, Bhutta MF, Butler CC, Holy C, Levine LH, Kvaerner KJ, Norman G,

2 Pennings RJ, Poe D, Silvola JT, Sudhoff H, Lund VJ. Eustachian tube dysfunction:

3 consensus statement on definition, types, clinical presentation and diagnosis. Clin

$4 \quad$ Otolaryngol. 2015;40:407-11.

5 11, McCoul ED, Anand VK. Eustachian tube balloon dilation surgery. Int Forum Allergy

$6 \quad$ Rhinol. 2012; 2:191-198.

Poistettu: 9

Poistettu: 0

7

8 
1 Table 1. Demographics.

2 Table 2. Current ear symptoms compared to the preoperative situation.

3 Table 3. Current overall ear symptoms compared to the preoperative situation.

4 Table 4. Disturbance caused by current ear symptoms.

5 Table 5. Willingness to undergo BET again if ear symptoms returned to the 6 preoperative level.

7 